\title{
La notion de handicap et ses transformations à travers les classifications internationales du handicap de l'OMS, 1980 et 2001
}

\section{Myriam Winance}

INSERM, U750-CERMES, Villejnif, France; CNRS, UMR8169-CERMES, Villejnif, France; EHESS, Paris, France, Université Paris XI, Paris. France; winance@vjf.cnrs.fr

Dynamis

[0211-9536] 2008; 28: 377-406
Fecha de recepción: 4 de septiembre de 2007

Fecha de aceptación: 21 de enero de 2008

SUMARIO: 1.-La Classification Internationale des Handicaps: Deficiences, incapacites, desavantages. 1.1.-Des causes aux conséquences des maladies. 1.2.-De la maladie à la personne malade. D'un point de vue médical à un point de vue social. 1.2.1.—La maladie. 1.2.2. - La déficience. 1.2.3.-L'incapacité. 1.2.4. - Le désavantage. 1.3.- La personne malade d'un point de vue social. 2.-De la $\mathrm{ClH}$ à la Classification Internationale du Fonctionnement, du handicap et de la santé. 2.1.-Controverse autour de la CIH. 2.2.—Les modèles alternatifs. 2.2.1.—Le modèle social du handicap. 2.2.2. - Le modèle interactif québécois. 2.2.3.-Les modèles alternatifs: des conséquences aux causes. 2.3.-Le processus de révision de la $\mathrm{ClH}$. 3.- La Classification Internationale du Fonctionnement, du handicap et de la santé. 3.1.-Des conséquences des maladies à la santé. 3.2.-Un modèle «biopsychosocial» pour décrire la santé, le handicap et le fonctionnement. 3.3.-Le handicap comme «défaut de fonctionnement». 4.-Conclusion.

RESUME: Dans cet article, l'auteur analyse l'évolution de la notion de handicap en s'intéressant aux deux classifications du handicap publiées par I'OMS, en 1980 puis en 2001. La «Classification Internationale du Handicap: déficiences, incapacités et désavantages. Un manuel de classification des conséquences des maladies», de 1980, prolonge la «Classification Internationales des Maladies»; le handicap y désigne les conséquences sociales des maladies. Dans la «Classification Internationale du Fonctionnement, du Handicap et de la Santé», publiée en 2001 , le handicap désigne un «défaut de fonctionnement» et est associé à la notion de santé. L'auteur s'intéresse à la question de la norme de référence utilisée dans les classifications pour définir le handicap.

MOTS CLEFS: Handicap, classification, OMS, santé, normes.

KEYWORDS: Disability, classification, WHO, health, norms. 
L'utilisation de la notion de handicap pour désigner les personnes atteintes de déficiences est récente. Elle émerge dans les pays occidentaux à la fin du 19ème siècle ${ }^{1}$ et se construit tout au long du 20ème siècle à la fois comme une catégorie administrative et comme une manière de se représenter et de traiter les personnes atteintes d'une déficience. Elle est liée à la mise en place progressive de l'Etat Providence et au processus de définition des droits sociaux ${ }^{2}$. En effet, suite au phénomène des accidents du travail à la fin du 19ème siècle (dans un contexte d'industrialisation), puis à la première guerre mondiale (dont de nombreux soldats reviennent mutilés), le dommage, qui se traduit par une incapacité de travail, est interprété comme le résultat d'une activité collective (l'activité industrielle ou la défense de la patrie). En tant que tel, il est considéré comme ouvrant droit à une réparation collective. Cette réparation a d'abord la forme d'une indemnisation ${ }^{3}$. Puis, suite à la première guerre mondiale et à la pénurie de travailleurs qui en résulte, une évolution s'opère. Réparer, c'est aussi compenser l'incapacité via la prothèse et les pratiques de réadaptation, pour permettre la réintégration du mutilé de guerre dans le monde du travail.

Les pratiques de réadaptation et de rééducation qui se développent tout au long du 20ème siècle sont, pour les personnes atteintes de déficiences, une alternative à l'assistance. Mais elles sont liées à un processus de normalisation en termes d'alignement sur la norme sociale de l'homme valide (travailler, être indépendant économiquement, avoir une famille). D'où une transformation des représentations et des définitions concernant les personnes atteintes de déficiences. Le terme «handicap» se substitue progressivement aux termes tels qu'invalides, infirmes, mutilés, idiots... et, durant les années 1970, s'impose comme catégorie unifiant l'ensemble des personnes atteintes d'une déficience, quelle que soit sa nature et son origine. Il met l'accent sur les conséquences sociales dues à la déficience, sur le désavantage qui en découle pour la personne. Il désigne l'écart à la norme sociale (celle de «l'homme moyen valide»), elle-même définie en termes de performances sociales, écart provoqué par la possession d'une déficience. Or,

1. Cooter, Roger. The disabled body. In: Cooter, Roger; Pickstone, John, eds. Medicine in the twentieth century. Amsterdam: Hartwood Academic Publishers; 2000, p. 367-383.

2. Ewald, François. L'Etat providence. Paris: Grasset; 1986.

3. En outre, elle ne concerne qu'une catégorie de personnes, les accidentés du travail puis les mutilés de guerre. Les autres personnes atteintes de déficiences, relèvent elles du champ de l'assistance (en France, ces personnes sont souvent placées dans des hospices). 
cette représentation des personnes porteuses de déficiences, couverte par la notion «handicap», ainsi que les pratiques et les politiques qui la concrétisent, ont conduit à une mise à l'écart des personnes handicapées, implicite ou explicite. Implicite, lorsque l'intégration des personnes handicapées se fait via l'obtention d'un statut particulier de «personne handicapée» qui la stigmatise. Explicite, lorsque l'intégration se fait via des établissements spécialisés (en France, ceux-ci connaîtront un important développement). D'où, une critique de ces représentations et pratiques, développée dans les années 1970 par les personnes handicapées elles-mêmes.

En effet, durant les années $1970^{4}$, les attentes des personnes handicapées évoluent, elles revendiquent le droit de participer à la vie sociale et de contrôler leur vie, elles contestent les pratiques et les politiques existantes qui les excluent au lieu de les inclure et font d'elles des sujets passifs soumis à la décision des professionnels. Elles critiquent la conception médicale et individuelle du handicap définissant celui-ci comme le résultat d'une déficience individuelle, devant être compensée. Elles s'opposent à la vision négative du handicap, faisant de celui-ci une tragédie personnelle. Dans les pays anglo-saxons, elles se rassemblent dans des mouvements et se mobilisent contre la discrimination, pour la défense de leurs droits, pour une société accessible et inclusive. Ces mouvements proposent des modèles alternatifs de définition du handicap, notamment le modèle social du handicap, base de leur action politique qui vise à transformer la société (et non plus à adapter l'individu) ${ }^{5}$. Ainsi, durant les années 1970 et 1980, sous l'impulsion d'acteurs de plus en plus divers, la notion de handicap telle qu'elle a été définie au cours du 20ème siècle, les pratiques et les politiques auxquelles elle est liée,

4. Cette période est par ailleurs une période d'évolution générale avec l'émergence de différents mouvements sociaux, un contexte social et économique favorable, etc... Au point de vue des théories sociales, cette période est également féconde. Les travaux de Foucault d'un côté, ceux de la sociologie de la déviance, de l'autre, seront appropriées par les activistes de différents mouvements (féminisme, gay, ethniques, handicap...) comme levier de transformation politique. Foucault, Michel. La volonté de savoir. Paris: Gallimard; 1976; Becker, Howard S. Outsiders; studies in the sociology of deviance. London: Free Press of Glencoe; 1963; Goffman, Erving. Stigma. Notes on the managements of spoiled identity. Harmondsworth: Pelican Book; 1968.

5. Barnes, Colin; Mercer, Geof; Shakespeare, Tom. Exploring disability. A sociological introduction. Cambridge: Polity Press; 1999. Oliver, Mike. Understanding disability. From theory to practice. London: Macmillan Press; 1996. Shakespeare, Tom, ed. The Disability reader. Cassell: London and New York; 1998. 
sont au centre de débats, internationaux et nationaux, qui conduisent à un éclatement des modèles de définition du handicap ${ }^{6}$.

Dans cet article, j'analyse cette évolution de la notion de handicap en me focalisant sur les deux classifications du handicap produites par l'OMS. En 1980, l'OMS publie une première classification en anglais sous le titre: International Classification of Impairments, Disabilities, and Handicaps. A manual of classification relating to the consequences of disease ${ }^{7}$, rapidement critiquée par les différents acteurs évoqués ci-dessus. D'où un long processus de révision, qui aboutit, en 2001, à un nouveau texte la Classification internationale $d u$ fonctionnement, $d u$ handicap et de la santé (International Classification of Functioning, Disability and Health), publiée en plusieurs langues (notamment anglais ${ }^{8}$, français ${ }^{9}$, espagnol ${ }^{10}$ ). L'intérêt de ces deux textes produits par l'OMS est de cristalliser chacun une conception du handicap, résultat d'une évolution historique. La première classification, publiée en 1980, se comprend dans la lignée du modèle réadaptatif qui s'est mis en place tout au long du 20ème siècle. La seconde, publiée en 2001, témoigne du débat et de l'évolution ayant eu lieu durant les 20 dernières années du siècle autour de la notion de handicap. Le changement de titre indique d'ailleurs l'un des déplacements majeurs qui s'est opéré en 20 ans: d'une description des conséquences des maladies, on est passé à une description de la santé. Le texte de 1980 liait la notion de handicap à celle de maladie, définissant le handicap en termes de conséquences sociales des maladies; le texte de 2001 lie la notion de handicap à celle de santé, et les définit en termes de fonctionnement. D'autres auteurs se sont intéressés aux classifications de l'OMS ${ }^{11}$, mais sans comparer véritablement les deux textes; mon objectif

6. Altman, M. Barbara. Disability definitions, models, classification schemes, and applications, In: Albrecht, L. Gary; Seelman, D. Katherine; Bury, Mike, eds. Handbook of disability studies. Thousand Oaks. London-New Delhi: Sages; 2001, p. 97-122.

7. WHO. International Classification of Impairments, Disabilities, and Handicaps. A manual of classification relating to the consequences of disease. Geneva: WHO; 1980.

8. WHO. International Classification of Functioning, Disability and Health. Geneva: WHO; 2001.

9. OMS. Classification internationale du fonctionnement, du handicap et de la santé. Genève: OMS; 2001.

10. Clasificación internacional del funcionamiento, de la discapacidad y de la salud. Madrid: OMSMinisterio de Asuntos Sociales (Instituto de Migraciones y Servicios Sociales; 2001.

11. Altman, n. 8. Chapireau, François. La nouvelle classification de I'OMS: Classification internationale du fonctionnement, du handicap et de la santé. Annales Médico-psychologiques, Revue Psychiatrique. 2002; 160 (3): 242-246; Chapireau, François. Environment in the international 
est, en me basant sur les textes des deux classifications, d'analyser ce déplacement opéré (d'une conception médicale à une conception plus large du handicap) qui me semble essentiel pour comprendre ce que recouvre aujourd'hui la notion de handicap. En d'autres termes, mon objectif est, en me focalisant sur ces deux textes, de faire apparaitre l'évolution qui s'est opérée en 30 ans. L'article sera divisé en trois parties. La première partie traite de la Classification internationale des handicaps: déficiences, incapacités, désavantages $(\mathrm{CIH})$, publiée en 1980 . La seconde partie retrace brièvement l'histoire du débat qui entoure cette classification entre 1980 et 2000. La dernière partie analyse la Classification Internationale du Fonctionnement, du handicap et de la santé (CIF), publiée en 2001.

\section{La Classification Internationale des Handicaps: Déficiences, inca- pacités, désavantages}

La première classification portant spécifiquement sur le handicap est publiée par l'OMS en 1980 en anglais. Son titre est International Classification of Impairments, Disabilities, and Handicaps. A manual of classification relating to the consequences of disease ${ }^{12}$. Elle est traduite en français en 1988, sous le titre Classification internationale des handicaps: déficiences, incapacités et désavantages. Un manuel de classification des conséquences des maladies ${ }^{13}$. Ce titre nomme l'objet de cette classification: elle concerne les conséquences des maladies, et se présente comme un outil complémentaire

Classification of functioning, disability and health. Journal of Intellectual Disability Research. 2004; 48 (4-5): 284; De Kleijn-de Vrankrijker, Marijke W. The long way from the International Classification of Impairments, Disabilities and Handicaps (ICIDH) to the International Classification of Functioning, Disability and Health (ICF). Disability and Rehabilitation. 2003; 25 (11-12): 561-564; Hurst, Rachel. The international disability right movement and the ICF. Disability and Rehabilitation. 2003; 25 (11-12): 572-576; Imrie, Robert. Demystifying disability: a review of the International Classification of Functioning, Disability and Health. Sociology of Health and IIIness. 2004; 26 (3): 287-305; Üstün, T. Bedirhan et al. The International Classification of Functioning, Disability and health: a new tool for understanding disability and health. Disability and Rehabilitation. 2003; 25 (11-12): 565-571.

12. $\mathrm{ClH}$ dans la suite du texte.

13. L'anglais utilise «handicap» pour désigner la troisième dimension, alors que le français utilise «désavantage» pour cette troisième dimension et «handicap» pour référer à l'ensemble des trois dimensions. 
de la Classification Internationale des Maladies ${ }^{14}$. «Les données contenues dans ce manuel se rapportent aux dimensions des expériences liées aux états de santé, complémentaires à celles du concept de maladie» ${ }^{15}$. Cette complémentarité est à la fois un prolongement et un déplacement par rapport au modèle de la CIM, la notion de maladie jouant le rôle d'articulation entre les deux classifications.

\subsection{Des causes aux conséquences des maladies}

L'objectif de l'OMS, à travers l'adoption de classifications internationales, est de disposer d'outils et de codes uniformisés structurant le recueil des informations nécessaires pour évaluer l'état de santé des populations et l'efficacité des services de santé. Les codes proposés par la CIM étaient ceux relatifs à la mortalité et à la morbidité. En effet, le concept central déployé dans la CIM est celui de maladie, définie comme le résultat d'une relation causale entre une atteinte organique et certaines manifestations pathologiques. L'enregistrement des informations relatives à la maladie et à son issue (guérison ou mort), suite au traitement médical de la cause, permet l'évaluation de l'efficacité des services de santé, car il rend visible le changement apparu dans l'état de la personne. Au niveau populationnel, les statistiques concernant la fréquence de la maladie, l'espérance de vie et les causes de décès, permettent une évaluation de la santé des populations.

Ces outils proposés dans la CIM ont rempli leur objectif tant que le principal problème de santé publique était le développement des maladies infectieuses et aiguës. Cependant, courant $20^{\text {ème }}$ siècle, le phénomène de la chronicité, c'est-à-dire de la persistance de manifestations liées soit à une maladie chronique, soit à une maladie ou un traumatisme passé, supplante

14. Concernant I'histoire de la Classification Internationale des Maladies (CIM), voir: Biraben, JeanNoël. Histoire des classifications de causes de décès et de maladies aux XVIIIème et XIXème siècles. In: Mensch und Gesundhait in der geschichte. Les hommes et la santé dans I'histoire. Berlin: Magghiesen Verlag; 1978. Bowker, C. Geoffrey; Star Leigh Star. Sorting things out. Classification and its consequences. Cambridge and London: The MIT Press; 1999; Roussel, Pascale. $\mathrm{ClH}-1 / \mathrm{ClH}-2$ : rénovation complète ou ravalement de façade? Handicap: Revue de sciences humaines et sociales. 1999; 81: 7-19.

15. OMS, Classification internationale des handicaps: déficiences, incapacités et désavantages. Un manuel de classification des conséquences des maladies. Paris: CTNERHI; 1993 (réédition). p. 4. 
celui des maladies infectieuses. Les codes proposés par la CIM ne suffisent plus pour décrire l'état de santé de l'individu, caractérisé par les séquelles; d'où la nécessité d'élaborer de nouveaux outils ${ }^{16}$. L'OMS confie à l'un de ses consultants, le Docteur Ph. Wood (rhumatologue, puis épidémiologiste), la tâche d'élaborer une classification des «conséquences des maladies»:

«La maladie empêche l'individu de s'acquitter de ses fonctions et de ses obligations courantes. Autrement dit, le malade est incapable de remplir son rôle habituel dans la société, et d'entretenir les rapports habituels avec autrui. Cette définition est assez large pour recouvrir la grande majorité des recours à un service de santé [...]. Bien que le modèle médical des maladies [...] constitue un instrument efficace pour aborder les troubles qui peuvent être traités ou guéris - la disparition des effets de la maladie étant un corollaire du traitement de la cause- il reste incomplet dans la mesure où il ne couvre pas les conséquences de la maladie. Or, ce sont ces dernières qui perturbent la vie quotidienne, et il faut disposer d'un cadre qui permettra de comprendre ces phénomènes, surtout en ce qui concerne les troubles chroniques et évolutifs ou irréversibles.

Il convient donc de développer la chaine des phénomènes liés à la maladie initiale, que l'on peut figurer ainsi: Maladie > déficience > incapacité $>$ désavantage» ${ }^{17}$.

Cette citation montre le déplacement qui s'opère dans la CIH, par rapport à la CIM. Ce qui pousse la personne à consulter un service de santé n'est pas sa maladie en tant que telle, mais le fait que sa maladie perturbe sa vie quotidienne, l'empêchant de tenir le rôle qui est le sien dans la société. D'un intérêt pour la maladie, on passe à un intérêt pour les effets de la maladie, effets décomposés en trois niveaux: la déficience, l'incapacité et le désavantage. D’où également, un élargissement de point de vue: s'intéresser aux conséquences de la maladie amène à s'intéresser non plus à la maladie, mais au «malade en société».

16. OMS, n. 15; Ravaud, Jean-François. Vers un modèle social du handicap. L'influence des organisations internationales et des mouvements de personnes handicapées. In: Riedmatten, R. D., ed. Une nouvelle approche de la différence. Genève: Médecine and Hygiène; 2001. p. 55-68; Ravaud, Jean-François; Mormiche, Pierre. Santé et handicaps, causes et conséquences d'inégalités sociales. Comprendre. 2003; (4): 87-106.

17. OMS, n. 15, p. 6-7. 


\subsection{De la maladie à la personne malade. D’un point de vue médical à un point de vue social}

Dans la CIH, centrée sur les conséquences de la maladie, la maladie ne s'analyse plus comme un processus causal autonome, mais comme touchant une personne, considérée comme un être social. La citation suivante confirme ce passage d'un point de vue médical sur la maladie à un point de vue social sur la «personne malade».

"Quand on observe les manifestations pathologiques, il est courant de se référer au concept de maladie. Dans cette notion et ce qui en dérive, par exemple la CIM, les phénomènes pathologiques sont considérés comme indépendants des individus chez lesquels ils apparaissent. [...] Les limites de ce mode d'approche sont pourtant assez évidentes: en isolant la maladie du malade, les conséquences de celle-ci sur celui-là risquent d'être négligées. Ces conséquences -qui sont les réponses du malade, ou des personnes qui l'entourent ou dont il dépend-prennent de plus en plus d'importance au fur et à mesure que le poids de la maladie s'accroît» ${ }^{18}$.

L'élargissement du point de vue, de la maladie au malade en société, se fait progressivement, par un processus qui s'enclenche à partir de la maladie, point d'articulation entre la CIM et la CIH. Le texte déploie les quatre niveaux successifs dans «l'expérience liée à l'état de santé», représentés par le schéma suivant:

\begin{tabular}{|c|c|c|c|c|c|c|}
\hline $\begin{array}{l}\text { Maladie ou } \\
\text { Trouble }\end{array}$ & $\rightarrow$ & $\begin{array}{l}\text { Déficience } \\
\text { (extériorisée) }\end{array}$ & $\rightarrow$ & $\begin{array}{l}\text { Incapacité } \\
\text { (objectivisée) }\end{array}$ & $\rightarrow$ & $\begin{array}{l}\text { Désavantage } \\
\text { (socialisée) }\end{array}$ \\
\hline $\begin{array}{l}\text { (situation } \\
\text { intrinsèque) }\end{array}$ & & & & (sic) & & \\
\hline
\end{tabular}

(OMS, 1993, p. 26). 


\subsubsection{La maladie}

Le premier niveau de l’expérience est la maladie.

«1. Quelque chose d'anormal se produit au niveau de l'individu (souligné dans le texte), soit à la naissance (phénomène congénital), soit plus tard (acquis). Une succession de circonstances causales, «étiologie», entraîne des modifications dans la structure ou le fonctionnement du corps, «la patho$\operatorname{logie} »{ }^{19}$.

La maladie est définie comme un processus causal interne et autonome par rapport à l'individu: la maladie transforme son corps, ensemble de structures et d'organes, sans que ce processus ne soit perceptible ni signifiant pour l'individu. Ce premier niveau de «l'expérience liée à l'état de santé» ne constitue donc pas, à proprement parler, un niveau d'expérience; la maladie, définie comme un événement «anormal», «une succession de circonstances causales», reste interne et non-perçue par l'individu. Le développement des conséquences de la maladie est le processus à travers lequel «l'état de santé» devient une expérience, perceptible et perçue par le malade ou son entourage. La maladie se développe en effet à travers un processus d'extériorisation, d'objectivation et de socialisation, ces trois dimensions constituant chacune un niveau de l'expérience. La maladie, phénomène interne et autonome, se transforme en un phénomène externe, objectif, puis social.

\subsubsection{La déficience}

L'extériorisation de la maladie réfère à la perception de ses manifestations par l'individu ou quelqu'un qui le côtoie.

«Quelqu'un prend conscience de ce fait. En d'autres termes, l'état pathologique est extériorisé. La plupart du temps, c'est le patient lui-même qui se rend compte des manifestations de la maladie, habituellement appelées «manifestations cliniques» [...]. En termes de comportement, l'individu est devenu, ou a été rendu, conscient du fait qu'il est en mauvaise santé. Sa maladie annonce les déficiences, anomalies de la structure ou de l'apparence du corps, ou de la fonction d'un organe ou d'un système, quelle qu'en soit la

19. OMS, n. 15, p. 22. 
cause. Les déficiences correspondent à des troubles au niveau de l'organe» 20 (souligné dans le texte).

Le deuxième niveau de l'expérience, la déficience, correspond à l'extériorisation de la maladie: l'individu prend conscience de sa maladie à travers ses manifestations, définies en termes d'anomalies et de troubles des organes ou des fonctions. La maladie, processus causal et interne, devient perceptible pour l'individu et son entourage car elle engendre des manifestations; d'un processus interne au corps, elle devient un processus externe qui modifie le corps de manière perceptible. Voici la définition donnée par la $\mathrm{CIH}$ de la déficience.

«Dans le domaine de la santé, la déficience correspond à toute perte de substance ou altération d'une structure ou fonction psychologique, physiologique ou anatomique. [...] La déficience correspond à une déviation par rapport à une certaine norme biomédicale de l'individu, et la définition de ses éléments doit être élaborée d'abord par les personnes qualifiées pour juger du fonctionnement physique et mental selon des normes généralement acceptées» ${ }^{21}$.

La déficience, comme la maladie dans la CIM, est définie en termes d'écart à la norme biomédicale, construite à travers l'histoire du savoir médical occidental $^{22}$ et connue par les spécialistes. La personne, si elle peut être celle qui perçoit les manifestations pathologiques, n'est pas reconnue comme compétente pour les évaluer. Elle est pour cela renvoyée devant les professionnels qui grâce à leurs connaissances, peuvent évaluer l'écart à la norme comme déficience.

\subsubsection{L'incapacité}

Le troisième niveau du processus est la traduction de la maladie sous forme d'incapacités à agir, traduction qui est interprétée comme un processus d'objectivation.

\footnotetext{
20. OMS, n. 15, p. 22.

21. OMS, n. 15, p. 23-24.

22. Augé, Marc; Herzlich, Claudine, eds. Le sens du mal: anthropologie, histoire, sociologie de la maladie. Paris: Ed. des Archives Contemporaines; 1983.
} 
«3. La capacité d'action ou le comportement de l'individu peuvent être altérés lorsque celui-ci est conscient de sa maladie, que cela en soit une conséquence directe ou que cela soit dû au fait qu'il se sait malade. Les activités habituelles risquent d'être réduites, et l'expérience de la maladie est ainsi objectivée. [...] Ces expériences correspondent aux incapacités qui reflètent les conséquences des déficiences en termes de performance fonctionnelle et d'activités de l'individu. Les incapacités correspondent à des troubles au niveau de la personne» 23 .

L'objectivation de la maladie réfère à la réduction concrète pour la personne, de ses capacités d'actions, dans sa vie quotidienne. La maladie se traduit par une diminution de sa performance et de ses activités normales.

«L'incapacité correspond à un écart par rapport à la norme en termes d'action de l'individu, et non pas d'un organe ou d'un mécanisme. Ce concept est caractérisé par un excès ou une diminution des comportements ou activités normales. Ces changements peuvent être temporaires ou permanents, réversibles ou non, progressifs ou régressifs. Leur caractéristique principale est l'objectivation. Cette dernière est le processus par lequel une limitation fonctionnelle s'exprime dans la réalité quotidienne, le problème étant objectivé par la gêne dans les activités du corps» 24 .

L'objectivation de la maladie correspond à la traduction de la maladie en une capacité d'action réduite et, comme pour la déficience, au processus de prise de conscience par l'individu de cette réduction de ses capacités. La définition de l'incapacité est la suivante.

«Dans le domaine de la santé, une incapacité correspond à toute réduction (résultant d'une déficience), partielle ou totale, de la capacité d'accomplir une activité d'une façon ou dans les limites considérées comme normales pour un être humain» 25 .

L'incapacité réfère à un écart à une norme définie en termes d'actions et de performances fonctionnelles ordinaires et moyennes, celles que chacun «normalement» possède. 


\subsubsection{Le désavantage}

Enfin, le dernier niveau, celui du désavantage, est la traduction de la maladie en un processus social.

«4. La perception de son incapacité, ou de l'altération du comportement ou du fonctionnement qui en découle, entraîne un désavantage pour l'individu atteint par rapport aux autres, faisant de son expérience un problème social, que cela soit une conséquence directe, ou que cela soit dû au fait qu'il se sait malade. Ce niveau reflète la réponse de la société à la situation de l'individu, qu'elle soit exprimée par une attitude (marginalisation ou stigmatisation par exemple), ou par un comportement (qui peut impliquer des instruments spécifiques, comme la législation). Ces expériences représentent le désavantage, résultant des déficiences et des incapacités. Ce niveau de conséquence des maladies est à l'évidence le plus problématique, étant donné son rapport explicite à la valeur attachée aux activités ou au statut de l'individu» ${ }^{26}$ (souligné dans le texte).

La notion de désavantage réfère au processus de socialisation de la maladie: à l'impact de la maladie pour la personne en tant qu'être social et à la traduction de la maladie en problème social. «Problème social» signifie ici un problème individuel dont le processus est social ou qui prend une ampleur sociale dans la mesure où il induit une réaction de la société.

Cette quatrième dimension du modèle, le désavantage social, constitue l'apport principal de la classification car elle introduit l'idée de conséquences sociales de la maladie.

«As we saw it, the World Health Organisation (WHO) was moving away from a narrow medical model of health and disease - one primarily concerned with body systems and aetiologies - to one which recognised the consequences of health-related phenomena. We were particularly concerned to argue for a clearer recognition of social disadvantage - the focus of the handicap codes in the classification ${ }^{27}$.

26. OMS, n. 15, p. 23.

27. Bury, Mike. A comment on the ICIDH2. Disability and Society. 2000; 15 (7): 1073. 
Comme l'indique M. Bury ${ }^{28}$, proche collaborateur de P. Wood, leur intention était de mettre l'accent sur l'importance sociale des maladies pour les personnes, importance sociale qui se manifeste par un désavantage, un handicap, pour la personne. Voici la définition du «désavantage social»: «Dans le domaine de la santé, le désavantage social pour un individu donné résulte d'une déficience ou d'une incapacité qui limite ou interdit l'accomplissement d'un rôle normal (en rapport avec lâge, le sexe, les facteurs sociaux et culturels)» ${ }^{29}$. La notion de désavantage social réalise le passage d'un point de vue biomédical à un point de vue social.

\subsection{La personne malade d'un point de vue social}

Le désavantage social ne concerne pas la personne en elle-même, mais la manière dont elle est socialement intégrée. Il concerne «la personne malade en société». «Les désavantages (...) se rapportent aux préjudices résultant pour l'individu de sa déficience ou de son incapacité; ils reflètent donc l'adaptation de l'individu et l'interaction entre lui et son milieu» ${ }^{30}$. Cette citation indique le double mécanisme du désavantage social, conséquence d'une inadéquation entre l'individu et son milieu (inadéquation analysée en termes d'adaptation), et d'un jugement négatif de la société concernant cette inadéquation. Premièrement, le désavantage social découle d'une comparaison entre l'individu et son milieu, plus précisément, entre l'individu et les attentes que son milieu formule à son égard, et du constat de l'existence d'une discordance entre les deux. «Le désavantage est caractérisé par une discordance entre l'activité de l'individu, ou son statut, et ce que son groupe d'appartenance attend de lui» ${ }^{31}$. En d'autres termes, l'individu, atteint d'une déficience ou d'incapacités, ne peut pas faire ce qu'en moyenne, les autres peuvent faire. La norme de référence est ici définie en termes de "performance moyenne». Deuxièmement, le désavantage découle d'un jugement de cet écart comme étant négatif et dévalorisant pour la personne. La notion de désavantage réfère à l'idée

28. L'un des acteurs ayant joué un rôle central dans la rédaction de la $\mathrm{ClH}$ est $\mathrm{M}$. Bury, sociologue de la médecine.

29. OMS, n. 15, p. 25

30. OMS, n. 15, p. 10.

31. OMS, n. 15, p. 26. 
d'un écart aux normes sociales, écart qui entraîne un discrédit pour la personne. Elle introduit explicitement la question de la valeur et du jugement comme élément constitutif du désavantage (et comme élément problématique).

«Un certain poids est attribué à la déviation de la norme structurelle, fonctionnelle ou d'exécution, soit par l'individu lui-même, soit par ses semblables dans le groupe auquel il appartient» ${ }^{32}$; ou «Le désavantage se détermine par rapport aux autres, de là, l'importance des valeurs sociales existantes qui, à leur tour, sont influencées par la trame institutionnelle. Ainsi, les attitudes et les réponses du non-handicapé jouent un rôle central dans le modelage de l'ego et la définition des possibilités pour un individu potentiellement désavantagé: ce dernier est très peu libre de déterminer ou de modifier sa propre réalité» ${ }^{33}$.

A travers cette notion de désavantage social, les auteurs de la classification reconnaissent l'importance du rôle des normes sociales dans le processus de production du handicap et intègrent l'idée de déviance issue des travaux des interactionnistes ${ }^{34}$, c'est-à-dire, de manière simplifiée, l'idée d'une qualification (positive ou négative) de l'individu en fonction de sa correspondance ou non-correspondance aux normes sociales, par les autres individus; la non-correspondance entraîne un discrédit (Goffman) ou un désavantage (OMS). En résumé, dans la $\mathrm{CIH}$, le handicap est défini comme l'écart à une norme sociale, définie en termes de performances moyennes, écart résultant d'une maladie et entraînant un jugement négatif, un discrédit, un désavantage pour la personne. En définissant le handicap de cette manière, la CIH apparaît comme l'aboutissement de l'histoire de la notion de handicap depuis la fin du 19ème siècle jusqu'aux années 1970.

\footnotetext{
32. OMS, n. 15, p. 25.

33. OMS, n. 15, p. 26.

34. Goffman, n. 4; Becker, n. 4; sur le lien entre les travaux de Goffman et l'histoire du «handicap», voir: Winance, Myriam. Being normally different? Changes to normalisation processes: from alignment to work on the norm. Disability and Society. 2007; 22 (6): 625-638.
} 


\section{De la CIH à la Classification Internationale du Fonctionnement, du handicap et de la santé}

\subsection{Controverse autour de la CIH}

Durant les années 1970-1980, le monde du handicap évolue. A la fin des années 70, les personnes handicapées se rassemblent dans des mouvements revendicatifs: aux Etats-Unis, le Mouvement pour la Vie Autonome ${ }^{35}$, en Grande-Bretagne, le Disability Movement ${ }^{36}$. L'association internationale des personnes handicapées (Disabled People International, DPI) est fondée en 1981. Les Nations Unies adoptent, en 1975, la Déclaration des droits des personnes handicapées, et promeuvent la pleine participation sociale et la non-discrimination des personnes handicapées. Les organisations internationales (OIT par exemple) et européennes ${ }^{37}$ produisent également des textes normatifs concernant les personnes handicapées. Ces évolutions influencent les politiques nationales, par exemple au Québec ${ }^{38}$, en France ${ }^{39}$. Dans ce contexte, éclate, à la fin des années 1980, une controverse internationale autour de la $\mathrm{CIH}$. Ces nouveaux acteurs réagissent à la $\mathrm{CIH}$. Dès sa parution, DPI rejette les définitions de l'OMS ${ }^{40}$. Les activistes des différents mouvements prennent position (voir infra) contre la CIH. Enfin, certains chercheurs, dont les chercheurs québécois (voir infra), demandent sa révision. Les critiques émises par ces acteurs convergent sur quatre points ${ }^{41}$.

35. Ravaud, n. 16; Williams, Gareth H. The movement for independent living: an evaluation and critique. Social Science and Medicine. 1984; 17 (15): 1003-1010.

36. Oliver, n. 5. Campbell, Jane. «Growing Pains» disability politics: The journey explained and described. In: Barton, Len; Oliver, Mike, eds. Disability studies: Past, present and future. Leeds: The Disability Press; 1997, p. 78-89.

37. Barral, Catherine. De l'influence des processus de normalisation internationaux sur les représentations du handicap. Handicap - Revue de sciences humaines et sociales. 1999; 81: 20-34.

38. Fougeyrollas, Pierre. L'évolution conceptuelle internationale dans le champ du handicap: Enjeux socio-politiques et contributions québécoises. PISTES. 2002; 4 (2)

39. Winance, Myriam; Ville, Isabelle; Ravaud, Jean-François. Disability policies in France: Changes and tensions between the category-based, universalist and personalized approaches. Scandinavian Journal of Disability Research. 2007; 9 (3): 160-181.

40. Enns, Henry. Déclaration de la Disabled Peoples'International (DPI) sur la classification internationale des déficiences, incapacités et handicaps $(\mathrm{CIDIH})$ de l'organisation mondiale de la santé (OMS). Janvier 1987. Réseau International CIDIH-Bulletin du CQCIDIH. 1989; 2 (2-3): 28-30.

41. Pour une revue des critiques, voir: Roussel, n. 14; Chapireau, François. Les débats et l'enjeu de la révision de la classification internationale des handicaps. Handicaps et Inadaptations-Les cahiers du CTNERHI. 1998; 79-80: 67-84; Chapireau, François; Colvez, Alain. Social disadvantage 
Première critique, la $\mathrm{CIH}$ incarne un modèle médical et individuel qui établit une relation de cause à effet entre la maladie, la déficience, les incapacités et le handicap, et qui situe l'origine du handicap exclusivement du côté de la personne. Celle-ci est handicapée parce qu'elle est malade. Elle se situe ainsi dans la ligne du modèle du handicap comme tragédie personnelle. Seconde critique, la $\mathrm{CIH}$ néglige l'importance des facteurs environnementaux (physiques, culturels, sociaux...) dans la production du handicap. Troisièmement, la terminologie employée par la CIH est négative et «handicapiste»; elle réfère aux manques de la personne et la dévalorise. Dernière critique, certains dénoncent le manque de cohérence de la $\mathrm{CIH}$, plus précisément, les chevauchements entre le niveau des incapacités et celui des désavantages. Si les critiques émises par ces divers acteurs se rejoignent, les modèles que chacun oppose à la CIH diffèrent. J'en évoquerai deux, importants pour comprendre le modèle proposé par la nouvelle classification: le modèle social développé par les activistes du «Disability Movement», et le modèle interactif développé par les Québécois ${ }^{42}$.

\subsection{Les modèles alternatifs}

\subsubsection{Le modèle social du handicap}

Le modèle social, ébauché à la fin des années 1970 et développé ensuite dans le cadre des Disability Studies ${ }^{43}$, redéfinit la causalité produisant le handicap. Lindividu porteur d'une déficience (impairment) n'est pas handicapé (disabled) à cause de cette déficience, mais à cause de la société, à cause des obstacles physiques, sociaux... qu'elle dresse à sa participation. Cet argument implique un modèle à deux pôles: la déficience (impairment) et le handicap (disability), et une séparation entre ces deux pôles. La déficience est une spécificité personnelle, elle désigne la description physique du corps, tandis

in the International Classification of Impairments, Disabilities and Handicaps. Social Science and Medicine. 1998; 47: 59-66.

42. Sur l'importance de ces deux modèles lors du processus de révision de la $\mathrm{ClH}$, voir par exemple: Bickenbach, Jérôme E. et al. Models of disablement, universalism and the international classification of impairments, disabilities and handicaps. Social Science and Medicine. 1999; 48: 1173-1187.

43. Albrecht, Gary L.; Ravaud, Jean-François; Stiker, Henri-Jacques. L'émergence des disability studies: état des lieux et perspectives. Sciences Sociales et Santé. 2001; 19 (4): 43-71. 
que le handicap est une réalité collective, il désigne l'exclusion sociale dont sont victimes les personnes atteintes d'une déficience du fait des multiples barrières dressées par la société à leur participation. La déficience est un problème médical, le handicap est une question politique ${ }^{44}$.

Le développement scientifique de ce modèle, durant les années 1980, a conduit à la construction d'une opposition forte entre ce modèle social et le modèle qualifié de médical et individuel, dont la CIH sera considérée comme l'archétype. D'où le positionnement des chercheurs anglais et nordaméricains, activistes issus du mouvement des personnes handicapées, contre la CIH. Lors du processus de révision de la $\mathrm{CIH}$, certains activistes, comme D. Pfeiffer, refuseront catégoriquement de participer ${ }^{45}$. D'autres, comme R. Hurst, accepteront de participer afin de transformer le modèle de la $\mathrm{CIH}^{46}$. Enfin, les responsables de la révision pour l'OMS, ne cesseront eux-mêmes de faire référence à ce modèle social ${ }^{47}$. Le modèle social, comme contre poids à la $\mathrm{CIH}$, jouera ainsi un rôle fondamental dans son processus de révision.

\subsubsection{Le modèle interactif québécois}

Les chercheurs québécois ${ }^{48}$ travaillent dans l'optique d'une modification de la $\mathrm{CIH}^{49}$. D'une part, ils cherchent à enclencher un processus de révision;

44. Barnes, Colin. Theories of disability and the origins of the oppression of disabled people in western society. In: Barton, Len, ed. Disability and Society. Emerging issues and insights. London-New York: Longman Sociology Series; 1996, p. 43-60; Barton, Len, ed. Disability and society: Emerging issues and insights. London-New York: Longman Sociology Series; 1996; Oliver, Mike. The politics of disablement. Basingstoke: Macmillan and St Martins Press; 1990.

45. Pfeiffer, David. The ICIDH and the need for its revision. Disability and Society. 1998; 13 (4): $503-$ 523; Pfeiffer, David. The devils are in the details: the ICIDH2 and the disability movement. Disability and Society. 2000; 15 (7): 1079-1082.

46. Hurst, n. 11. Hurst, Rachel. To revise or not to revise? Disability and Society. 2000; 15 (7): 10831087.

47. OMS, n. 9; Bickenbach, n. 42. Bickenbach, Jérôme.E.; Shroot, R.L. Le modèle social du handicap et la révision de la classification internationale des handicaps (CIDIH). Handicaps et Inadaptations - Les cahiers du CTNERHI. 1998; 79-80: 35-48.

48. Travaillant d'abord dans le cadre de l'Office des Personnes Handicapées du Québec (créé en 1978), puis rassemblés dans le Comité québécois sur la CIH, créé en 1986.

49. Fougeyrollas, n. 38; Fougeyrollas, Pierre. Les déterminants environnementaux de la participations sociale des personnes ayant des incapacités: le défi sociopolitique de la révision de la CIDIH. Canadian journal of rehabilitation. 1997; 10 (2): 147-160; Fougeyrollas, Pierre. La classification 
d'autre part, ils développent une classification alternative à la $\mathrm{CIH}$, le «Processus de production des handicaps», publiée en $1991^{50}$. Cette classification présente un modèle interactif du handicap (par opposition au modèle linéaire de la $\mathrm{CIH}^{51}$ ), en reprenant certains des concepts utilisés dans la $\mathrm{CIH}$ (celui de déficience et d'incapacité), mais surtout, en introduisant les notions de facteurs environnementaux et d'habitudes de vie. Les facteurs environnementaux désignent l'ensemble des dimensions sociales, culturelles, écologiques qui déterminent l'organisation et le contexte d'une société ${ }^{52}$. La notion d'habitudes de vie est définie ainsi:

«Les habitudes de vie sont celles qui assurent la survie et l'épanouissement d'une personne dans sa société tout au long de son existence. Ce sont les activités quotidiennes et domestiques ainsi que les rôles sociaux valorisés par le contexte socio-culturel pour une personne selon son âge, son sexe et son identité sociale et personnelle» ${ }^{53}$.

Défini avec ces concepts, le handicap est le résultat d'une interaction entre la personne et son environnement, physique ou social, et se traduit par une difficulté à accomplir ses habitudes de vie, en fonction de son âge, son sexe...

Comme dans la $\mathrm{CIH}$, le handicap est l'impossibilité de remplir les attentes normatives sociales (cfr. définition de la notion «d'habitudes de vie») et cette impossibilité est liée à l'état corporel de l'individu. Cependant, ce lien n'est plus interprété comme un lien causal direct et linéaire. Au contraire, le lien observé entre déficiences, incapacités et difficultés à accomplir les habitudes de vie est à expliquer. D'où un renversement: les auteurs ne déploient plus une relation allant de la cause (la maladie) à ses conséquences (le handicap), mais une relation allant des conséquences (le handicap) à ses

québécoise du processus de production du handicap et la révision de la CIDIH. Handicaps et Inadaptations-Les cahiers du CTNERHI. 1998; 79-80: 84-103; Ravaud, Jean-François; Fougeyrollas, Pierre. La convergence progressive des positions franco-québécoises. Santé, Société et Solidarité. 2005; 2: 13-27.

50. Le processus de production des handicaps. Réseau International CIDIH-Bulletin du CQCIDIH. 1991; 4 (3).

51. Et en se distinguant du modèle social qui est unidimensionnel, ne reconnaissant qu'une causalité sociale.

52. Fougeyrollas, Pierre. Une autre façon de le dire. Réseau International CIDIH-Bulletin du CQCIDIH. 1990; 3 (1): 15.

53. Fougeyrollas, n. 52, p. 14. 
causes (multiples et agissant en interaction). L'impossibilité d'accomplir les habitudes de vie est analysée comme résultant de l'interaction entre la dimension corporelle et la dimension environnementale, physique ou sociale, (jouant le rôle «d'obstacle» ou «de facilitateur»). L'écart à la norme attendue et valorisée n'est plus rapporté à la personne, mais à une situation interactive. Ce modèle met donc en évidence l'idée que la norme sociale est relative à une interaction et la normalité (ou l'écart à cette normalité, le handicap) construite dans cette interaction. Pour réduire l'apparition de handicaps (comme écarts à ce qui est normalement attendu et valorisé), il faut non seulement adapter la personne, mais surtout travailler sur les attitudes, sur l'environnement ${ }^{54}$, il faut travailler sur l'interaction et sur les normes qui la sous-tendent.

\subsubsection{Les modèles alternatifs: des conséquences aux causes.}

Les deux modèles alternatifs apportent deux modifications essentielles par rapport au modèle de la CIH. Premièrement, la référence à la norme biomédicale s'efface devant celle à la norme sociale. Les difficultés quotidiennes rencontrées par les personnes présentant une spécificité corporelle constituent le point de départ de leur construction conceptuelle et de leur définition du handicap. Dans les deux cas, la personne handicapée est d'emblée une personne en société. L'univers de référence n'est plus celui de la maladie (CIM), ni du malade en société (CIH), mais celui de la personne en société et de la vie quotidienne. Deuxièmement, les deux modèles, de manière différente, reviennent à la question de la causalité (centrale dans la CIM), mais en prenant comme point de départ, le point d'arrivée de la $\mathrm{CIH}$ : le handicap. Ils reconnaissent que celui-ci coexiste avec une déficience, mais nient qu'il soit causé uniquement par cette déficience. En partant du constat que les personnes porteuses de déficiences sont désavantagées, handicapées, ils redéfinissent, chacun à leur manière, la causalité produisant ce désavantage ou handicap et aboutissent à des causalités sociales. Le déplacement opéré est important. D'une analyse des conséquences sociales de la maladie, on passe à une analyse des causalités sociales du handicap.

54. Fougeyrollas, n. 52, p. 13. 


\subsection{Le processus de révision de la $\mathrm{CIH}^{55}$}

En 1993, l'OMS reconnaît la CIH comme une classification officielle de l'OMS et s'engage dans un processus de révision qui débute, véritablement, en 1995, suite à l'implication des Etats-Unis (porteur d'une volonté politique et de moyens financiers) et à un changement politique interne à l'OMS (changement de responsable). Des centres collaborateurs pour l'OMS sont créés pour l'Australie (1995), le Royaume-Uni, le Japon et les Pays Nordiques (1998), attestant un élargissement international du processus (qui reste cependant occidental); des groupes de travail sont organisés (facteurs environnementaux, santé mentale, enfance). Une large consultation internationale a lieu, impliquant des professionnels médicaux, des responsables administratifs et politiques, des chercheurs, des associations de personnes handicapées ${ }^{56}$. De juin 96 à décembre 2000, 6 versions provisoires sont rédigées et testées. Ce processus aboutit à un texte, la Classification Internationale du Fonctionnement, du Handicap et de la Santé ${ }^{57}$, adopté par l'assemblée générale de l'OMS au printemps $2001^{58}$. Ce texte, complexe, est le résultat des négociations entre des acteurs devenus multiples, et donc, des compromis entre leurs différentes perspectives. Dans la section suivante, je m'intéresse à la définition du handicap contenue dans cette classification et à ses évolutions par rapport à la $\mathrm{CIH}$.

\section{La Classification Internationale du Fonctionnement, du handicap et de la santé}

\subsection{Des conséquences des maladies à la santé}

Le changement de titre de la classification indique le déplacement majeur qui s'est opéré entre les deux classifications. La CIH avait pour objectif

55. Pour une synthèse de ce processus, voir: Fougeyrollas, n. 38; Barral, Catherine; Roussel, Pascale. De la CIH à la CIF. Le processus de révision. Handicap-Revue de sciences humaines et sociales. 2002; 94-95: 1-24. Fougeyrollas et Barral retracent, chacun de leur point de vue, I'histoire du processus de révision.

56. Ainsi, la liste des remerciements de la CIF (p. 265-278) est beaucoup plus fournie que la liste de la $\mathrm{ClH}$.

57. CIF dans la suite du texte.

58. OMS, n. 9; OMS, n. 10. 
d'analyser les conséquences des maladies. L'objectif de la CIF est de décrire la santé:

«La CIF s'est éloignée d'une classification des «conséquences de la maladie» (version 1980) pour devenir une classification des «composantes de la santé». Les «composantes de la santé» définissent ce qui constitue la santé, alors que les «conséquences»se focalisent sur l'impact de la maladie ou tout problème de santé qui peut en résulter. Ainsi, la CIF adopte une position neutre par rapport à l'étiologie et permet aux chercheurs d'inférer les causes des situations qu'ils observent à l'aide des méthodes scientifiques appropriées» ${ }^{59}$.

Le point de départ de la CIH était la maladie, le handicap étant finalement défini comme «l'expression sociale de la maladie». Dans la CIF, le point de départ de la réflexion est la santé, définie en termes de fonctionnement (aspects positifs de la santé) et de handicap (aspects négatifs de la santé). La maladie ne disparaît pas du modèle, mais elle ne sert plus de point de référence pour définir le handicap.

En effet, le handicap est conceptualisé comme une dimension de la santé humaine et comme une modalité particulière du fonctionnement humain, lorsque celui-ci est problématique. Cette conception est confirmée par les responsables du processus de révision de la $\mathrm{CIH}$, dans un article daté de 1999 "Disablement is now understood as an identifiable variation of human functioning.» ${ }^{60}$. D'où, également, l'optique universaliste de la CIF, le handicap, en tant que modalité de fonctionnement, concerne tout un chacun. «Un malentendu largement répandu consiste à penser que la CIF ne concerne que les personnes handicapées: en fait, elle concerne tout un chacun» ${ }^{61}$. On observe ici l'influence forte du Mouvement pour la Vie Autonome, qui défend cette approche universaliste, contre une approche en termes de droits d'une minorité. Les outils élaborés dans la CIF n'ont pas pour but la caractérisation d'une population, «les personnes handicapées», mais la description du fonctionnement, dans ses aspects positifs et/ou négatifs (et on parle, dans ce dernier cas, de handicap), de toute personne. La CIF introduit donc un déplacement central par rapport

\footnotetext{
59. OMS, n. 9, p. 4.

60. Bickenbach, n. 42, p. 1184

61. OMS, n. 9, p. 7.
} 
à la CIH. Celle-ci s'intéressait au «malade en société», la CIF s'intéresse à la personne, quelle qu'elle soit, et à son état de santé, indépendamment d'une relation effective avec un service de santé. Le point de vue adopté par la CIF a changé. En ce sens, la CIF a retenu les apports des modèles alternatifs. Elle ne déploie plus un processus causal, partant d'une cause, la maladie, à ses conséquences, le handicap, mais elle propose des outils pour décrire la vie quotidienne des personnes, leur bon ou mauvais fonctionnement.

En même temps, et ce point est essentiel, il s'agit de décrire le fonctionnement et le handicap, lorsque ceux-ci sont liés à un problème de santé ${ }^{62}$.

«Les informations codées se situent toujours dans le contexte d'un problème de santé. Bien qu'il ne soit pas nécessaire, pour utiliser un code, d'établir formellement le lien entre un problème de santé et les aspects du fonctionnement et du handicap qui feront l'objet du codage, la CIF n'en reste pas moins une classification relative à la santé. Elle présuppose donc l'existence d'un problème de santé quelconque.» ${ }^{63}$; ou, «The ICIDH-2, like its predecessor, is a classification of consequences of health conditions. [...] It provides the essential language and structure for scientific investigations of the consequences of health conditions in the human population» ${ }^{64}$.

La CIF, comme la CIM et la CIH, s’intéresse aux conséquences des «états de santé», compris comme ensemble de problèmes dus à un trouble ou une maladie. Elle présuppose et affirme un lien entre handicap et problème de santé. Cependant, en réponse aux critiques faites à la $\mathrm{CIH}$, le lien établi entre handicap et problème de santé n'est pas un lien causal, mais un lien de coexistence, de présence simultanée. On retrouve donc la conceptualisation du handicap en termes de conséquences d'un problème de santé, mais celui-ci n'est pas considéré, de manière a priori, comme cause du handicap, d'où l'affirmation d'une position neutre par rapport à l'étiologie (voir citation supra). La CIF ne définit pas, de manière a priori, une causalité du handicap, mais présente un modèle interactif et multidimensionnel,

62. Point contesté par les tenants du modèle social qui récusent que le handicap puisse être interprété comme un problème de santé.

63. OMS, n. 9, p. 234.

64. Bickenbach, n. 42, p. 1184 
qualifié de modèle «biopsychosocial», qui pourra être utilisé pour analyser ses causalités.

Le modèle de définition du handicap présenté dans la CIF se situe dans la continuité des précédentes classifications (CIM et CIH), tout en suivant certaines évolutions opérées par les modèles alternatifs. Son point de départ, comme celui de ces modèles alternatifs, est élargi; il s'agit de décrire l'état de santé des personnes (ayant des troubles de santé), définie en termes de fonctionnement ou de difficultés de fonctionnement (handicap), en explorant ses différentes facettes, biologique, psychologique et sociale, et en ne s'interrogeant sur les causalités que dans un second temps. Par là, la CIF ne présente plus la santé comme l'objectif des services de soins, mais comme un état des personnes, état dont il faut analyser les différentes dimensions. Mais la CIF se situe en continuité avec la CIM et la CIH, en réaffirmant le lien entre handicap et problème de santé, précisé en termes de trouble ou de maladie. On retrouve ici la conception biomédicale de la santé. Cette tension entre deux conceptions de la santé traverse la CIF; celle-ci développe une conception large de la santé, en termes de fonctionnement et de handicap, grâce à un modèle «biopsychosocial», tout en gardant, à l'intérieur de son modèle, la conception restreinte de la santé comme état physiologique ou pathologique.

\subsection{Un modèle «biopsychosocial» pour décrire la santé, le handicap et le fonctionnement}

L'opposition entre le modèle médical et le modèle social, et le débat avec le modèle interactif québécois traversent le modèle biopsychosocial de la CIF. D'une part, les auteurs de la CIF ne pouvaient ignorer l'approche sociale du handicap. D'autre part, travaillant dans le cadre de l'OMS et en continuité par rapport aux classifications antérieures, ils étaient porteurs d'une approche biomédicale de la santé. Comme le montre la citation suivante, tiré d'un texte postérieur à la CIF, ils devaient tenir les deux pôles de l'opposition.

«Le handicap est un phénomène complexe qui est à la fois un problème au niveau du corps de la personne et un phénomène complexe et principalement social. Le handicap est toujours le résultat d'une interaction entre les caractéristiques individuelles et les caractéristiques du contexte global 
dans lequel la personne vit, mais certains aspects du handicap sont presque entièrement internes à la personne, alors que d'autres lui sont totalement externes» 65 .

Dans cette citation, l'OMS reconnaît la complexité du handicap, problème individuel, qui concerne le corps, et problème social, qui concerne la société. Elle reconnaît également la dimension interactive du handicap, défendue par les Québécois. Mais, en dernier lieu, elle décompose le handicap, en distinguant des aspects internes et des aspects externes à la personne.

Dans la classification, cette complexité du handicap se traduit par un modèle (représenté par le schéma suivant) qui, premièrement, analyse le fonctionnement et le handicap comme le résultat d'une interaction entre un problème de santé et des facteurs contextuels (personnels ou environnementaux). Deuxièmement, ce modèle reprend et transforme les trois niveaux de l'expérience du handicap distingués dans la CIH: «Ces domaines [de la santé et domaines connexes de la santé] peuvent être décrits en prenant comme

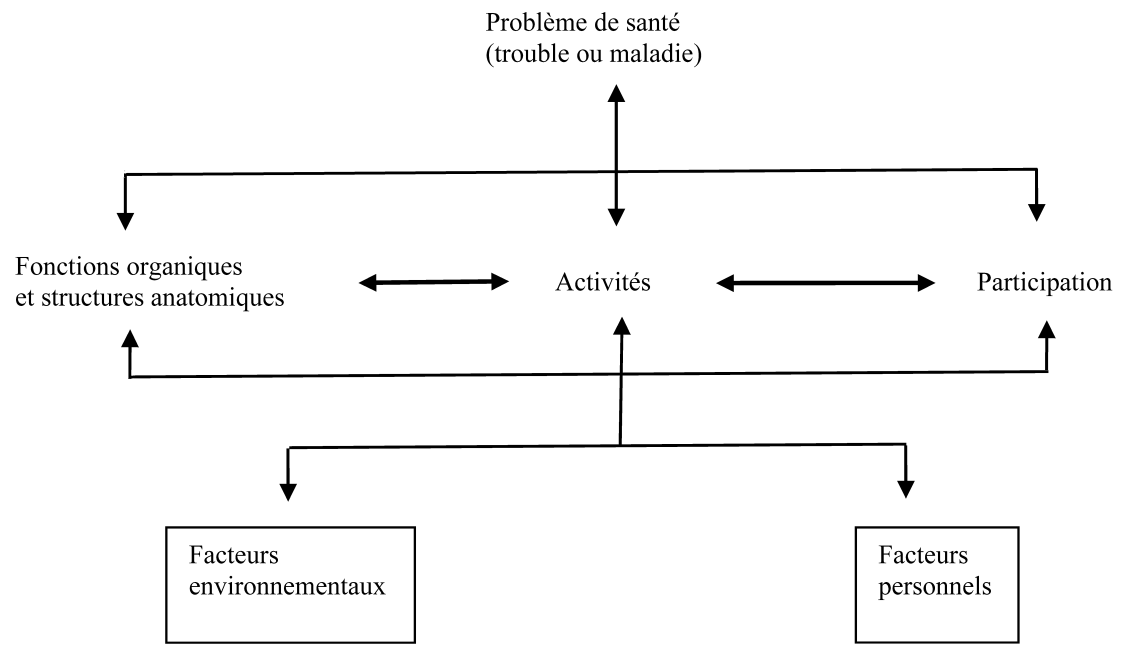

(OMS, 2001, p. 19)

65. OMS, Vers un langage commun pour le fonctionnement, le handicap et la santé. CIF: Classification internationale du fonctionnement, du handicap et de la santé. Handicap-Revue de sciences humaines et sociales. 2002; 94-95: p. 32. 
perspectives l'organisme, la personne en tant qu'individu ou la personne en tant qu'être social, selon deux listes de base: 1) les fonctions organiques et les structures anatomiques; 2) les activités et la participation» ${ }^{66}$. La classification distingue ce qui concerne le fonctionnement biomédical (du corps - «aspect interne du handicap») et ce qui concerne le fonctionnement social (de l'individu ou de la personne comme être social - «aspect externe du handicap»).

La lecture verticale du schéma propose les outils permettant aux utilisateurs d'analyser le processus de handicap ou du fonctionnement comme un processus interactif entre trois dimensions: un problème de santé, des facteurs personnels et des facteurs environnementaux. La première dimension est définie de la manière suivante:

«Problème de santé est une expression générique désignant une maladie (aiguë ou chronique), un trouble, une lésion ou un traumatisme. Ce terme peut également faire allusion à d'autres situations telles que la grossesse, le vieillissement, le stress, une anomalie congénitale ou une prédisposition génétique. Les problèmes de santé sont codés à l'aide de la CIM-10» ${ }^{67}$.

Dans ce passage, le lien entre la CIF et la CIM est affirmé, même si la CIF n'est plus présentée comme développant le processus causal lié à la maladie. Les facteurs personnels, non classifiés dans la CIF ${ }^{68}$, sont définis de la manière suivante: «Les facteurs personnels sont des facteurs contextuels qui ont trait à l'individu tels que l'âge, le sexe, la condition sociale, les expériences de la vie, etc., qui ne sont pas classifiés dans la CIF mais que les utilisateurs peuvent intégrer à leurs applications de la CIF» ${ }^{69}$. Enfin, les facteurs environnementaux qui font eux l'objet d'une classification sont définis de la manière suivante: «Les facteurs environnementaux constituent une composante de la CIF et renvoient à tous les aspects du monde extérieur ou extrinsèque qui forment le contexte de la vie d'un individu et, à ce titre, ont une incidence sur le fonctionnement de celui-ci» ${ }^{70}$. Ces facteurs environnementaux peuvent jouer le rôle de facilitateurs et amé-

\footnotetext{
66. OMS, n. 9, p. 3.

67. OMS, n. 9, p. 222.

68. Avec comme justification de cette non-classification, leur variabilité culturelle.

69. OMS, n. 9, p. 223.

70. OMS, n. 9, p. 224.
} 
liorer le fonctionnement de la personne, ou être des obstacles et entraver le fonctionnement de la personne.

La lecture horizontale du schéma permet la description du fonctionnement et du handicap à l'aide de trois composantes qui à la fois reprennent et remplacent les trois dimensions de l'expérience développées dans la CIH. La première composante est la composante corporelle qui comprend le fonctionnement des systèmes organiques et des structures anatomiques. La deuxième composante est la composante individuelle, nommée «activité» et définie comme «l'exécution d'une tâche ou d'une action par un individu» ${ }^{71}$. Enfin, la troisième composante est la composante sociale, couverte sous la notion de «participation» et définie comme «l'implication de l'individu dans une situation de vie réelle». ${ }^{72}$

Ce schéma, dans sa double lecture, verticale et horizontale, renforce la conception large de la santé, en explicitant les définitions du fonctionnement et de son contraire, le handicap, comme états multidimensionnels, physiologique, individuel et social, résultant d'une interaction non prédéfinie entre un problème de santé, des facteurs personnels et des facteurs environnementaux.

«Fonctionnement est un terme générique couvrant les fonctions organiques, les structures anatomiques, les activités et la participation. Il désigne les aspects positifs de l'interaction entre un individu (ayant un problème de santé) et les facteurs contextuels face auxquels il évolue (facteurs personnels et environnementaux). Et Handicap est un terme générique désignant les déficiences, les limitations d'activité et les restrictions de participation. Il désigne les aspects négatifs de l'interaction entre un individu (ayant un problème de santé) et les facteurs contextuels face auxquels il évolue (facteurs personnels et environnementaux)» ${ }^{73}$.

71. OMS, n. 9, p. 223.

72. Notons cependant qu'il n'existe pas dans la classification deux listes différentes pour les activités et la participation, mais une liste unique pour ces deux dimensions. La distinction entre les deux niveaux se fait lors de l'utilisation concrète de la CIF, en fonction de la situation locale. Cette décision d'adopter une liste unique pour les deux dimensions, activités et participation, a fait l'objet de critiques lors du processus de révision. La manière dont elle est présentée dans le schéma (distinction de deux dimensions) et le texte (liste unique) est l'exemple typique des compromis réalisés lors de la consultation.

73. OMS, n. 9, p. 223-224. 
Dans ce cadre, la notion de handicap, opposée à celle de fonctionnement, ne désigne plus les conséquences sociales de la maladie, mais un défaut de fonctionnement, lié d'une manière ou d'une autre, à un problème de santé. Dans la CIH, la personne handicapée était d'abord définie dans sa relation avec un système de soin, comme un malade (écart à une norme biomédicale); cette maladie se traduisant dans la sphère sociale par certaines difficultés (écart à une norme sociale, en termes de réduction de capacités et de performances). Dans la CIF, être handicapé, c'est ne pas fonctionner ou du moins, «mal» fonctionner, et cela dans le cadre d'une interaction entre la personne et son contexte. Or, cette définition du handicap repose sur une transformation de la norme de référence, par rapport à celle qui sous-tendait la $\mathrm{CIH}$.

\subsection{Le handicap comme "défaut de fonctionnement»}

Dans la $\mathrm{CIH}$, la question de la norme était traitée en deux étapes. Première étape, la déficience était définie par rapport à une norme biomédicale, l'incapacité par rapport à une norme d'action et le désavantage par rapport à une norme de performance sociale, le point de référence étant ce qu'en moyenne, l'individu valide est capable de faire. Mais, deuxième étape, la dimension du désavantage comprenait l'idée d'un jugement de valeur (négatif et dévalorisant) posé par la société sur la manière dont la personne accomplissait son rôle. Dans la CIF, on retrouve la première étape. Le fonctionnement est évalué par rapport à une norme biomédicale et à une norme d'actions. La définition de ces deux normes est proche de celles données dans la CIH. Pour la norme biomédicale, on retrouve la référence au savoir médical.

«Les déficiences représentent des écarts par rapport à certaines normes généralement acceptées de l'état biomédical du corps et de ses fonctions. La définition de leurs composantes repose principalement sur le jugement des personnes compétentes pour évaluer le fonctionnement physique et mental par rapport à des normes généralement reconnue» ${ }^{74}$.

74. OMS, n. 9, p. 12-13. 
Pour la norme d'action, on retrouve la comparaison avec les personnes valides:

«Des difficultés ou des problèmes peuvent survenir dans ces domaines quand intervient une modification qualitative ou quantitative de la manière dont les fonctions sont exécutées. Les limitations et les restrictions sont estimées par rapport à une norme généralement acceptée dans une population donnée. La norme par rapport à laquelle on évalue la capacité et la performance d'une personne donnée est la capacité ou la performance d'une personne qui ne présente pas le même problème de santé (maladie, trouble ou lésion)» ${ }^{75}$.

Si l'on retrouve l'idée de comparaison entre individus ayant des troubles de santé et ceux n'en ayant pas, l'idée de jugement de valeur contenue dans la notion de désavantage n'est pas reprise dans la CIF. Cependant, une autre norme est développée dans la CIF avec les deux notions (présentes dans les citations ci-dessus) de performance et de capacité. Ces deux notions sont utilisées comme code qualificatif pour évaluer le niveau d'activité et de participation de la personne. Or, ils introduisent l'idée d'un écart interne à l'individu, alors que la notion de désavantage comprenait l'idée d'un écart externe (produit par un jugement social).

Voici la manière dont la CIF définit les notions de performance et de capacité:

«Le code qualificatif de performance décrit ce qu'un individu fait dans son environnement ordinaire. Du fait que l'environnement ordinaire comprend un contexte sociétal, la performance peut donc aussi être perçue comme une «implication dans une situation de vie réelle» ou comme «l' expérience vécue» de personnes considérées dans leur cadre de vie réel. Ce contexte comprend les facteurs environnementaux, c'est-à-dire tous les aspects du monde physique, du contexte social et des attitudes, et peut faire l'objet d'un codage en utilisant la liste des facteurs environnementaux. Le code qualificatif de capacité décrit l'aptitude d'un individu à effectuer une tâche ou à mener une action. Ce schéma est conçu pour indiquer le niveau de fonctionnement le plus élevé possible qu'une personne est susceptible d'atteindre dans un domaine donné à un moment donné. Pour évaluer l'aptitude réelle d'une personne, on devrait pouvoir disposer d'un environnement «normalisé», qui neutraliserait les influences variables d'environnements différents sur chaque

75. OMS, n. 9, p. 16 
personne. (...) Ainsi, la capacité reflète l'aptitude d'une personne ajustée des facteurs environnementaux. (...) Lécart entre capacité et performance reflète la différence d'impacts entre environnement usuel et environnement standard. Elle constitue ainsi un guide utile pour déterminer ce qui peut être modifié dans le cadre de vie de la personne concernée pour améliorer son niveau de réalisation ${ }^{76}$.

Cette citation est intéressante car elle introduit l'idée d'un écart entre ce que la personne fait et ce qu'elle pourrait faire en fonction de son environnement. Autrement dit, l'écart n'est plus entre la personne ayant un trouble de santé et celle qui n'en a pas, mais devient interne à la personne, la norme devenant le niveau de fonctionnement le plus élevé que cette personne puisse atteindre dans un environnement «normalisé». En outre, cette citation montre un report de l'action de normalisation de l'individu sur l'environnement, mais la classification ne donne pas les outils permettant de définir cet environnement «normalisé» et «normalisant».

En modifiant ainsi les normes de références, la CIF place au centre de la définition du handicap, la question de la déficience et celle de l'action, et fait passer à l'arrière plan, la question du jugement social. Le handicap, défaut de fonctionnement, réfère aux déficiences de la personne et/ou à ses difficultés à agir ou à participer qu'elle rencontre. Cette conception du handicap s'inscrit dans la conjoncture actuelle qui met l'accent sur la notion de participation et sur la figure d'une personne citoyenne qui agit. Alors que la $\mathrm{CIH}$ analysait le handicap comme le résultat d'un jugement social variable en fonction des sociétés et en ce sens reconnaissait une extériorité (par rapport à la classification) de la norme de référence, la CIF semble balancer entre deux positions. D'un côté, elle semble avoir internalisé la norme de référence sous la forme d'une injonction à la participation (participer et agir sont le signe d'un bon fonctionnement, d'une bonne santé). D'un autre côté, en définissant le handicap comme le résultat d'une interaction et en reconnaissant le rôle de l'environnement, elle semble tendre vers la reconnaissance d'une relativité de la norme et d'une possibilité de la définir en situation et selon cette situation. 


\section{Conclusion}

S'intéresser aux deux textes produits sur le handicap par l'OMS à 20 ans d'intervalle -20 ans durant lesquels le paysage politique du handicap a fondamentalement bougé- permet de saisir certaines des évolutions qui ont eu lieu durant cette période. Le modèle de définition de la CIH, en définissant le handicap comme le désavantage social dû à une maladie, était l'aboutissement de l'histoire du handicap telle qu'elle s'est déployée au cours du $20^{\text {ème }}$ siècle. Durant les années 1980 et 2000, les personnes handicapées contestent cette définition, ainsi que les pratiques et les politiques qui lui sont liées. Elles veulent participer à la vie ordinaire et mettent l'accent sur les obstacles environnementaux et sociaux qui les en empêchent. Corrélativement, elles redéfinissent le handicap comme la conséquence d'un processus d'exclusion sociale. Le texte de la CIF est le reflet de ces oppositions entre ces nouveaux acteurs et leurs modèles. Il témoigne de la transformation du contexte et des modèles.

Le but de la CIF est, par rapport à celui de la CIH, élargi; la CIF n'a plus seulement pour finalité le recueil des informations statistiques nécessaires à l'évaluation de la santé comprise comme objectif des services de soins, mais la description de la santé à des fins statistiques, de recherche, clinique, de politique sociale, pédagogique... Pour cela, elle propose une définition du handicap en termes de fonctionnement, définition qui se veut universelle. En détachant la notion de handicap de celle de maladie et en l'attachant à celle de santé, la CIF élargit son point de vue: elle s'intéresse à l'état de santé de la personne, de toute personne, et cherche à en décrire les différentes facettes, biologique, psychologique et sociale. La définition $\mathrm{du}$ handicap comme variation du fonctionnement humain ouvre des perspectives intéressantes, la difficulté étant cependant de trouver les outils permettant de décrire cette variation sans en définir de manière apriori les caractéristiques. Ainsi, la liste définissant l'activité et la participation, telle qu'elle est actuellement constituée dans la CIF, semble trop occidentalocentrée pour permettre cette possibilité. 